PROCEEDINGS OF THE

AMERICAN MATHEMATICAL SOCIETY

Volume 126, Number 2, February 1998, Pages 333-342

S 0002-9939(98)04110-0

\title{
PSEUDOCHARACTERS ON FREE SEMIGROUPS INVARIANT WITH RESPECT TO THEIR AUTOMORPHISM GROUPS
}

\author{
V. A. FAĬZIEV \\ (Communicated by Palle E. T. Jorgensen)
}

\begin{abstract}
Let $\mathcal{F}$ be a free semigroup and let $A$ be an automorphism group of $\mathcal{F}$. A description is given of the space of real functions $\varphi$ on semigroup $\mathcal{F}$ satisfying the following conditions:

1) the set $\{\varphi(x y)-\varphi(x)-\varphi(y) ; x, y \in \mathcal{F}\}$ is bounded;

2) $\varphi\left(x^{n}\right)=n \varphi(x)$ for any $x \in \mathcal{F}$ and $n \in N$;

3) $\varphi\left(x^{\tau}\right)=\varphi(x) \quad \forall x \in \mathcal{F}$, and $\forall \tau \in A$.
\end{abstract}

\section{INTRODUCTION}

The question 'if we replace a given functional equation by a functional inequality, then under what conditions will the solutions of the inequality be close to the solutions of the equation' was posed for the functional equation $f(x y)=f(x) \cdot f(y)$ for $x, y$ in a group $G$ in [1] in connection with the results of the papers [2]-[4].

For a mapping $f$ of the group $G$ into the semigroup of linear transformations of a vector space, in the papers [5], [6] sufficient conditions for the coincidence of the solution of the functional inequality $\|f(x y)-f(x) \cdot f(y)\|<c$ with the solution of the corresponding functional equation $f(x y)-f(x) \cdot f(y)=0$ was studied. In the papers [7]-[9], it was independently shown that if a continuous mapping $f$ of a compact group $G$ into the algebra of endomorphisms of a Banach space satisfies the relation $\|f(x y)-f(x) \cdot f(y)\| \leqslant \delta$ for all $x, y \in G$ with a sufficiently small $\delta>0$, then it is $\varepsilon$-close to a continuous representation $g$ of the same group in the same Banach space (i.e., we have $\|f(x)-g(x)\|<\varepsilon$ for all $x \in G)$. In the paper [9], for any positive $\varepsilon$, an example is constructed of a matrix-valued mapping $f$ of the group $G$ with generators $a, b, c$, and $d$ and defining relation $a^{-1} b^{-1} a b c^{-1} d^{-1} c d=1$, such that $f$ satisfies the relation $\|f(x y)-f(x) \cdot f(y)\|<\varepsilon$ but is not $\delta$-close $(0<\delta<1 / 10)$ to any representation of the group $G$.

In the papers [10]-[13] the following new algebraic object was introduced.

Definition 1. A pseudocharacter of a semigroup $S$ is a real function $\varphi$ on $S$ satisfying the following conditions:

1) the set $\{\varphi(x y)-\varphi(x)-\varphi(y) ; x, y \in S\}$ is bounded;

2) $\varphi\left(x^{n}\right)=n \varphi(x)$ for any $x \in S$ and $n \in N$.

Received by the editors December 19, 1995 and, in revised form, June 26, 1996 and July 26, 1996.

1991 Mathematics Subject Classification. Primary 20M15, 20M30.

Key words and phrases. Semigroup, free semigroup, group, semidirect product, function, endomorphism, automorphism, pseudocharacter, character, linear space.

(C)1998 American Mathematical Society 
The set of pseudocharacters of a semigroup $S$ is a vector space (with respect to the usual operations of addition of functions and their multiplication by numbers), which will be denoted by $P X(S)$. The subspace of $P X(S)$ consisting of real additive characters of the semigroup $S$ will be denoted by $X(S)$. The notion of pseudocharacter permits us to understand the nature of the existence of mappings satisfying the relation of the form $\|f(x y)-f(x) \cdot f(y)\|<\varepsilon$ without being close to any representation: if $\varphi$ is a pseudocharacter and $|\varphi(x y)-\varphi(x)-\varphi(y)|<c$, then for any $\alpha \in R$ the function $f(x)=\exp (i \alpha \varphi(x)), x \in G$, satisfies the relation $|f(x y)-f(x) \cdot f(y)|<|\exp (i \alpha c)-1|$ for $|\alpha c|<\pi$. Thus, for any $c>0$ and for any $\varepsilon>0$, the value $|f(x y)-f(x) \cdot f(y)|$ can be considered to be uniformly smaller than $\varepsilon$ for a suitable choice of $\alpha>0$.

In [12]-[17] a description of the spaces of pseudocharacters on free groups and semigroups, semidirect and free products of semigroups was given.

The referee of this paper pointed out to the following connection of pseudocharacters with the theory of Banach algebra cohomology. The condition (1) of the definition of pseudocharacter exactly gives a bounded 2-cocycle on $S$. Hence, if semigroup $S$ has a nontrivial pseudocharacter, i.e., $P X(S) \backslash X(S) \neq \emptyset$, then arguing as $\left[18\right.$, Proposition 2.8] we obtain $H^{2}(S, \mathbb{C}) \neq 0$.

Definition 2. A pseudocharacter $f$ of a semigroup $S$ is said to be invariant with respect to a semigroup $K$ of endomorphisms of $S$ if for any elements $x \in S$ and any $\tau \in K$ the relation $f\left(x^{\tau}\right)=f(x)$ holds.

Let us denote the space of pseudocharacters of semigroup $S$ that are invariant with respect to a semigroup $K$ of its endomorphisms by $P X(S, K)$. Denote by $X(S, K)$ the subspace of $P X(S, K)$ consisting of real additive characters of $S$.

In [15] the following result was obtained.

Theorem 1. $P X(K \cdot S)=P X(K) \dot{+} P X(S, K)$, where $K \cdot S$ denotes the semidirect product of the semigroups $K$ and $S$ and $K$ acts on $S$ by endomorphisms.

In connection with this result, the problem of describing the space of pseudocharacters of some semigroup $S$ which are invariant with respect of some semigroup $K$ of its endomorphisms arises.

\section{$\S 1$. The MAIN RESUlts}

The aim of this article is to give a description of the space of pseudocharacters of a free semigroup invariant with respect to some group of its automorphisms.

From Definition 1 it follows that if $\mathcal{F}$ is a free semigroup of rank 1 , then $P X(\mathcal{F})=$ $X(\mathcal{F})$. Therefore, in the sequel we shall assume that $\mathcal{F}$ is a free semigroup with the set $X$ of free generators, and that the cardinality of $X$ is greater than 1 . Let $A$ be a subgroup of $A u t \mathcal{F}$.

Definition 3. Two elements $u, v$ of $\mathcal{F}$ are said to be conjugate if there are $a$ and $b$ in $\mathcal{F}$ such that $u=a b, v=b a$. The relation of conjugacy is denoted by $\sim$. We will say that elements $u$ and $v$ of $\mathcal{F}$ are $A$-conjugate if there is an $a \in A$ such that $u^{a} \sim v$

Definition 4. An element of $\mathcal{F}$ is called simple if it is not a nontrivial power of another element. The set of the simple elements of the semigroup $\mathcal{F}$ will be denoted by $\mathcal{P}$. 
It is easy to see that the relation of $A$-conjugacy is an equivalence relation on $\mathcal{F}$ and on $\mathcal{P}$. We denote the relation of $A$-conjugacy by $\sim_{A}$.

Definition 5. Let $v=x_{1} \cdot x_{2} \ldots x_{n}, \quad x_{i} \in X$. The number $|v|=n$ is called the length of the element $v$.

Now for each word $v$ in the semigroup $\mathcal{F}$ we introduce the set of "beginnings" $H(v)$ and the set of "ends" $K(v)$ as follows. If $v \in X$, we put $H(v)=K(v)=\varnothing$. If $v=x_{i_{1}} \cdot x_{i_{2}} \cdots \cdots x_{i_{n}}, n>1$, where $x_{i j} \in X$, we set

$$
\begin{aligned}
& H(v)=\left\{x_{i_{1}}, x_{i_{1}} x_{i_{2}}, \ldots, x_{i_{1}} x_{i_{2}} \cdots x_{i_{n-1}}\right\}, \\
& K(v)=\left\{x_{i_{2}} \cdots x_{i_{n}}, x_{i_{3}} \cdots x_{i_{n}}, \ldots, x_{i_{n-1}} x_{i_{n}}, x_{i_{n}}\right\} .
\end{aligned}
$$

Lemma 1. There is a set $P$ of representatives of conjugacy classes in $\mathcal{P}$ such that for each $w \in P H(w) \cap K(w)=\varnothing$.

Proof. See Lemma 8 in [17].

For any element $w$ of $\mathcal{F}$ such that $H(w) \cap K(w)=\varnothing$ in [17] the functions $\eta_{w}$ and $e_{w}$ were defined as follows: if $v \in \mathcal{F}$, then $\eta_{w}(v)$ is equal to the number of occurrences of $w$ in the word $v ; e_{w}=\max \left\{\eta_{w}\left(v^{\prime}\right), v^{\prime} \sim v\right\}$.

Lemma 2. Let $w \in P$. Then the function $e_{w}$ is a pseudocharacter and we have the following relations:

1) $\left|e_{w}(u v)-e_{w}(u)-e_{w}(v)\right| \leqslant 2$ for any elements $u$ and $v$ of $\mathcal{F}$;

2) if $|u|<|w|$, then $e_{w}(u)=0$;

3) if $|u|=|w|$, then $e_{w}(u)=1$ for $u \sim w$ and $e_{w}(u)=0$ for $u$ not conjugate to $w$.

Proof. See Lemma 13 in [17].

Let $u \sim v$. It is easy to verify that for any $a \in A$ the relation $u^{a} \sim v^{a}$ holds. For an arbitrary element $w$ of $\mathcal{F}$ we define the sets $S t(w), B(w), C(w), A(w), D(w)$ as follows. Let $S t(w)$ denote the stabilizer of element $w$ in group $A$. Let $B(w)$ denote the stabilizer of the class of conjugacy elements containing $w$ in group $A$, i.e., $b \in B(w)$ if and only if $w^{b} \sim w$. It is evidently that $S t(w)$ and $B(w)$ are subgroups of group $A$ and $S t(w) \subseteq B(w)$. Let $C(w)$ (respectively $A(w)$ ) be a system of representatives of left cosets of the group $B(w)$ (respectively $A$ ) by subgroup $S t(w)$, i.e., $B(w)=S t(w) \cdot C(w), \quad A=S t(w) \cdot A(w)$. By $D(w)$ we denote the system of representatives of left cosets of the group $A$ by the subgroup $B(w)$. Then we have $A=B(w) \cdot D(w)$. For any $v \in \mathcal{F}$ denote by $M(v)$ the set of values of the function $a \rightarrow v^{a}, \quad a \in A$. For each element $w$ of the semigroup $\mathcal{F}$ such that $H(w) \cap K(w)=\varnothing$ let us define functions $\delta_{w}$ and $\bar{\delta}_{w}$ as follows: $\delta_{w}(v)=\sum_{t \in M(w)} e_{t}(v) ; \quad \bar{\delta}_{w}(v)=\frac{1}{|C(w)|} \delta_{w}(v)$.

From [17, Theorem 1] we obtain that the functions $\delta_{w}$ and $\overline{\delta_{w}}$ are pseudocharacters of the semigroup $\mathcal{F}$.

Lemma 4. For each $v$ in $\mathcal{F}$ and $a$ in $A$ the equalities $\delta_{w}\left(v^{a}\right)=\delta_{w}(v) ; \bar{\delta}_{w}\left(v^{a}\right)$ $=\bar{\delta}_{w}(v)$ is valid, i.e., the pseudocharacters $\delta_{w}$ and $\bar{\delta}_{w}$ are invariant relative to the action of the group $A$.

Let $x \in \mathcal{F}$; we set $\bar{H}(x)=H(x) \cup\{x\}$ and $\bar{K}(x)=K(x) \cup\{x\}$. For each pair of elements $x, y$ of $\mathcal{F}$ we define measures $\mu_{x, y}, \mu_{x, y, x}$, and $\nu_{x, y}$ on $P$ as follows. 
We set $\mu_{x, y}(w)=1$ if there exist $a$ and $b$ such that $a \in \bar{K}(x), b \in \bar{H}(y)$, and $w=a b$; otherwise we set $\mu_{x, y}(w)=0$.

We set $\mu_{x, y, x}(w)=1$ if there exist $c$ and $d$ such that $c \in \bar{K}(x), d \in \bar{H}(x)$, and $w=c y d ;$ otherwise we set $\mu_{x, y, x}(w)=0$.

Finally, we set $\nu_{x, y}(w)=\mu_{x, y}(w)+\mu_{y, x}(w)+\mu_{x, y, x}(w)+\mu_{y, x, y}(w)-\mu_{x, x}(w)-$ $\mu_{y, y}(w)$.

Definition 6. For any pair $u, v \in \mathcal{F}$ we define measures $\omega_{u, v}^{A}(w)$ and $\bar{\omega}_{u, v}^{A}(w)$ on the set $T$ as follows: $\omega_{u, v}^{A}(w)=\sum_{t \in M(w)} \nu_{u, v}(t), \quad \bar{\omega}_{u, v}^{A}(w)=\frac{1}{|C(w)|} \cdot \omega_{u, v}^{A}(w)$.

Let us fix some system of representatives $T$ of the classes of $A$-conjugate elements lying in $\mathcal{P}$ such that $H(w) \cap K(w)=\varnothing$ for any $w \in T$. Denote by $T_{n}$ the subset of $T$ consisting of elements of length $n$. Denote by $\operatorname{ZPX}(\mathcal{F}, A)$ the subspace of $P X(\mathcal{F}, A)$ consisting of the pseudocharacters of semigroup $\mathcal{F}$ vanishing on $T_{1}$.

Denote by $L^{\prime}(T)$ the space of real functions $\alpha$ on $T$ satisfying the following conditions :

1) $\left.\alpha\right|_{T_{1}} \equiv 0$

2) $\left.\alpha\right|_{T_{n}}$ is bounded for each positive integer $n$;

3) the set $\left\{\int_{T} \alpha d \bar{\omega}_{x, y}^{A}, \quad x, y \in \mathcal{F}\right\}$ is bounded.

Now we can give the statements of the main results.

Theorem 2. Let $\gamma$ be a bounded function on the set $T_{n}$; then the function $\psi_{\gamma}=$ $\sum_{w \in T_{n}} \gamma(w) \bar{\delta}_{w}$ belongs to the space $\operatorname{PX}(\mathcal{F}, A)$ and $\psi_{\gamma}(w)=\gamma(w) \quad \forall w \in T_{n}$.

Theorem 3. Each element $\varphi$ of the space $Z P X(\mathcal{F}, A)$ is uniquely representable in the form $\varphi=\sum_{w \in T} \alpha(w) \bar{\delta}_{w}, \quad \alpha \in L^{\prime}(T)$.

Lemma 9. The expression $\sum_{t \in T_{1}} \gamma(t) \bar{\delta}_{t}$ is the general form of an element of the space $X(\mathcal{F}, A)$, where $\gamma$ is an arbitrary function from $T_{1}$ to $\mathbb{R}$.

Corollary 1. Each element $f$ of the space $\operatorname{PX}(\mathcal{F}, A)$ is uniquely representable in the form $f=\chi+\sum_{w \in T} \alpha(w) \delta_{w}, \quad \chi \in X(\mathcal{F}, A), \quad \alpha \in L^{\prime}(T)$.

\section{$\S 2$. Proofs of the main Results}

Lemma 3. For any element $a \in A$ the following equalities hold: $a^{-1} B(w) a=$ $B\left(w^{a}\right) ; a^{-1} H(w) a=H(w)^{a}$.

Proof. The proof is straightforward.

This lemma implies that the mapping $\widehat{a}: b \rightarrow a^{-1} b a$ establishes a one-to-one correspondence between $B(w)$ and $B\left(w^{a}\right)$, and between $S t(w)$ and $S t\left(w^{a}\right)$ too. Hence, the sets $C(w)$ and $C\left(w^{a}\right)$ have equal cardinality. For each $\gamma \in C(w)$ the word $w^{\gamma}$ is a cyclic permutation of word $w$. Let $\gamma_{1}$ and $\gamma_{2}$ be distinct elements of $C(w)$; then $w^{\gamma_{1}} \neq w^{\gamma_{2}}$. In fact if $w^{\gamma_{1}}=w^{\gamma_{2}}$, then $\gamma_{2}^{-1} \gamma_{1} \in S(w)$ and there is $h \in S t(w)$ such that $\gamma_{1}=\gamma_{2} \cdot h$. Hence, the elements $\gamma_{1}, \gamma_{2}$ belong to the same coset of $B(w)$ by $S(w)$. This means that $\gamma_{1}=\gamma_{2}$ and we obtain a contradiction to the assumption. Thus the mapping $w \rightarrow w^{\gamma}$ is an embedding of $C(w)$ into the set of distinct elements of $\mathcal{F}$ which are conjugated with $w$. Therefore, $|C(w)| \leq|w|$. Let $a_{1}, a_{2}$ be elements of $A(w)$ such that $a_{1} \neq a_{2}$. It can easily be checked that $w^{a_{1}} \neq w^{a_{2}}$. Let $M(w)=\left\{w^{a} ; a \in A(w)\right\}$. It is clear that $M(w)$ coincides with the set of values of the function $a \rightarrow w^{a}, \quad a \in A$. It is evident that for any $a \in A$ and any $w \in \mathcal{F}$ the equality $(M(w))^{a}=M(w)$ holds. 
Lemma 4. For each $v$ in $\mathcal{F}$ and each $a$ in $A$ the equalities $\delta_{w}\left(v^{a}\right)=\delta_{w}(v)$, $\bar{\delta}_{w}\left(v^{a}\right)=\bar{\delta}_{w}(v)$ are valid; i.e., the peudocharacters $\delta_{w}$ and $\bar{\delta}_{w}$ are invariant relative to the action of the group A.

Proof. In [17, Lemma 11] it was shown that for each $w \in \mathcal{F}$ such that $H(w) \cap K(w)$ $=\varnothing$ we have the equality

$$
e_{w}(v)=\eta_{w}(v)+\mu_{v, v}(w) .
$$

It is easy to verify that for any $u, v$ from $\mathcal{F}$ the equalities $\eta_{w}\left(v^{a}\right)=\eta_{w^{a^{-1}}}(v)$ and $\mu_{v, u}\left(w^{a}\right)=\mu_{v^{a^{-1}}, u^{a}-1}(w) \quad$ hold. Now from (1) we obtain the equality

$$
e_{w}\left(v^{a}\right)=e_{w^{a-1}}(v) \text {. }
$$

Further, we have

$$
\begin{aligned}
\delta_{w}\left(v^{a}\right) & =\sum_{t \in M(w)} e_{t}\left(v^{a}\right)=\sum_{t \in M(w)} e_{t^{a}-1}(v) \quad \text { by } \\
& =\sum_{u \in M^{a-1}(w)} e_{u}(v)=\sum_{t \in M(w)} e_{t}(v)=\delta_{w}(v) .
\end{aligned}
$$

The lemma is proved.

Lemma 2 implies that $\delta_{w}(w)$ is equal to the number of elements in the set $M(w)$ which conjugate with $w$. Therefore, $\delta_{w}(w)=|C(w)|$ and $1 \leq \delta_{w}(w) \leq|w|$ and we have $\bar{\delta}_{w}(w)=1$. It is clear that a class of $A$-conjugate elements containing $t$ consists of a union of classes of conjugate elements such that their representatives are elements $t^{\gamma}, \gamma \in D(t)$. Let $\gamma_{1}, \gamma_{2}$ be different elements of $D(t)$. It is clear that $w^{\gamma_{1}} \nsim w^{\gamma_{2}}$. By Lemma 1 in every class of $A$-conjugate elements belonging to $\mathcal{P}$ there is an element $w$ such that the relation $H(w) \cap K(w)=\varnothing$ holds.

Let us fix some system of representatives $T$ of classes of $A$-conjugate elements lying in $\mathcal{P}$ and having the property $H(w) \cap K(w)=\varnothing$. Evidently, every element $\varphi$ from the space $\operatorname{PX}(\mathcal{F}, A)$ is defined by its restriction to $T$.

Lemma 5. Let $\gamma$ be an arbitrary function on $T_{1}$. Then the function

$$
\psi_{\gamma}=\sum_{w \in T_{1}} \gamma(w) \bar{\delta}_{w}
$$

belongs to the space $X(\mathcal{F}, A)$ and $\left.\psi\right|_{T_{1}}=\gamma$.

Proof. The proof is by direct calculation.

Let $\operatorname{PX}\left(\mathcal{F}_{T_{1}}, A\right)$ be the subspace of $\operatorname{PX}(\mathcal{F}, A)$, consisting of pseudocharacters of the semigroup $\mathcal{F}$ vanishing on the set $T_{1}$. Suppose $\varphi \in P X(\mathcal{F}, A)$ and $\gamma=\left.\varphi\right|_{T_{1}}$. Then the function $f=\varphi-\sum_{w \in T_{1}} \gamma(w) \delta_{w}$ belongs to the space $\operatorname{PX}(\mathcal{F}, A)$ and $\left.f\right|_{T_{1}} \equiv 0$.

Thus the problem of a description of space $\operatorname{PX}(\mathcal{F}, A)$ is reduced to the problem of a description of the space $\operatorname{PX}\left(\mathcal{F}_{T_{1}}, A\right)$.

Definition 6. For any pair $u, v \in \mathcal{F}$ we define measures $\omega_{u, v}^{A}(w)$ and $\bar{\omega}_{u, v}^{A}(w)$ on the set $T$ as follows : $\omega_{u, v}^{A}(w)=\sum_{t \in M(w)} \nu_{u, v}(t), \quad \bar{\omega}_{u, v}^{A}(w)=\frac{1}{|C(w)|} \cdot \omega_{u, v}^{A}(w)$.

From Lemma 2 we obtain

Lemma 6. For any $v \in \mathcal{F}$ and any $w \in T$ the following relations hold: 1) $\bar{\delta}_{w}(v)=$ 0 if $|v|<|w|$; 2) $\bar{\delta}_{w}(v)=0$ if $|v|<|w|$ and $v \chi_{A} w$; 3) $\bar{\delta}_{w}(w)=1$. 
Lemma 7. For each $u, v \in \mathcal{F}$ and each $w \in T$ we have $\bar{\omega}_{u, v}^{A}(w)=\bar{\delta}_{w}(u v)-\bar{\delta}_{w}(u)-$ $\bar{\delta}_{w}(v)$.

Proof. By Lemma 12 from [17] we have

$$
\nu_{u, v}(t)=e_{t}(u v)-e_{t}(u)-e_{t}(v) .
$$

Therefore, $\bar{\omega}_{u, v}^{A}(w)=\frac{1}{C(w)} \sum_{t \in M(w)}\left[e_{t}(u v)-e_{t}(u)-e_{t}(v)\right]=\bar{\delta}_{w}(u v)-\bar{\delta}_{w}(u)-\bar{\delta}_{w}(v)$. The lemma is proved.

Theorem 2. Let $\gamma$ be a bounded function on the set $T_{n}$; then the function

$$
\psi_{\gamma}=\sum_{w \in T_{n}} \gamma(w) \bar{\delta}_{w}
$$

belongs to the space $\operatorname{PX}(\mathcal{F}, A)$ and $\psi_{\gamma}(w)=\gamma(w) \quad \forall w \in T_{n}$.

Proof. Consider the measures $\nu_{u, v}$. Let $M_{n}$ be the subset of $\mathcal{F}$ consisting of the words of length $n$ in the alphabet $X$. Then for each $w \in T_{n} \quad M(w)$ is a subset of $M_{|w|}$. From the definition of the measures $\nu_{u, v}$ it follows that for any words $u$ and $v$ and $n \geq 2$ the estimate $\left|M_{n} \cap \operatorname{supp} \nu_{u, v}\right| \leq 4(n-1)$ holds. Note that the collection $\bigcup_{w \in T_{n}}\{t ; t \in M(w)\}$ consists of pairwise distinct elements. In fact, it is clear for elements belonging to $\{t ; t \in M(w)\}$. Now suppose that $t_{1}=t_{2}$, $t_{1} \in M\left(w_{1}\right), \quad t_{2} \in M\left(w_{2}\right), \quad w_{1} \chi_{A} w_{2}$. Since $t_{1} \sim_{A} w_{1}$, and $t_{2} \sim_{A} w_{2}$ we obtain that $w_{1} \sim_{A} w_{2}$, and we come to a contradiction to the assumption. Hence, there exist at most $4(n-1)$ elements $w$ of $M_{n}$ such that $\omega_{u, v}^{A}(w) \neq 0$. Therefore, $\left|T_{n} \cap \operatorname{supp} \omega_{u, v}\right| \leq 4(n-1)$ and

$$
\left|T_{n} \cap \operatorname{supp} \bar{\omega}_{u, v}^{A}\right| \leq 4(n-1) .
$$

From (4) and Lemma 2 we obtain $\left|\nu_{u, v}(w)\right| \leq 2$; therefore,

$$
\left|\omega_{u, v}^{A}(w)\right| \leq \sum_{t \in M(w)}\left|\nu_{u, v}(t)\right| \leq 2 \cdot\left|T_{n} \cap \operatorname{supp} \nu_{u, v}\right| \leq 2 \cdot 4(n-1),
$$

and $\left|\bar{\omega}_{u, v}^{A}(w)\right| \leq 8(n-1)$. Let $c>0$ be such that $|\gamma(w)| \leq c$ for all $w \in T_{n}$; then $\left|\frac{\gamma(w)}{C(w)}\right| \leq c$. Hence, we have

$$
\begin{aligned}
& \left|\psi_{\gamma}(u v)-\psi_{\gamma}(u)-\psi_{\gamma}(v)\right|=\left|\sum_{w \in T_{n}} \gamma(w)\left[\bar{\delta}_{w}(u v)-\bar{\delta}_{w}(u)-\bar{\delta}_{w}(v)\right]\right| \\
& \quad=\left|\sum_{w \in T_{n}} \gamma(w) \bar{\omega}_{u, v}^{A}(w)\right| \\
& \quad \leq c \cdot \sum_{w \in T_{n}}\left|\bar{\omega}_{u, v}^{A}(w)\right| \leq c \cdot 8(n-1) \cdot 4(n-1)=32 c(n-1)^{2} \quad(\text { by } \quad(6),(7)) .
\end{aligned}
$$

Now from Lemma 6 it follows that $\psi_{\gamma}(w)=\gamma(w) \quad \forall w \in T_{n}$. The theorem is proved.

Denote by $K$ the set of functions $\varphi$ on the semigroup $\mathcal{F}$ satisfying the following conditions:

1) $\varphi\left(x^{n}\right)=n \varphi(x) \quad \forall n \in N, \quad \forall x \in \mathcal{F}$;

2) $\varphi(x y)=\varphi(y x) \quad \forall x, y \in \mathcal{F}$ 
3) $\left.\varphi\right|_{P_{1}} \equiv 0,\left.\quad \varphi\right|_{P_{i}} \quad$ is bounded $\forall i \in N$;

4) $\varphi\left(x^{a}\right)=\varphi(x) \quad \forall x \in \mathcal{F}, \forall a \in A$.

It is evident that $K$ is a linear space relative to the ordinary operations. Denote by $Z \operatorname{PX}(\mathcal{F}, A)$ the subspace of $\operatorname{PX}(\mathcal{F}, A)$ consisting of pseudocharacters of the semigroup $\mathcal{F}$ vanishing on $T_{1}$ (and hence on the set $P_{1}=X$ too). It is clear that $Z P X(\mathcal{F}, A)$ is a subspace of $K$.

Lemma 8. Let $\varphi \in P X(\mathcal{F}, A)$ and $\left.\varphi\right|_{T_{1}} \equiv 0$. Then the function $\varphi$ is bounded on the set $T_{n} \quad \forall n \in N$.

Proof. The proof is by induction on $n$.

Denote by $L(T)$ the space of real functions $\alpha$ on $T$ satisfying the following conditions: $\left.\alpha\right|_{T_{1}} \equiv 0$, and $\left.\alpha\right|_{T_{n}}$ is bounded for each positive integer $n$. It is obvious that $L^{\prime}(T)$ is a subspace of $L(T)$. Let us construct an isomorphism $\theta$ between the spaces $K$ and $L(T)$. Let $\varphi \in K$. For each $i \in N$ we define the function $\alpha_{i}: T_{i} \rightarrow R$ by induction as follows: let $\alpha_{1} \equiv 0$, and if the values $\alpha_{1}, \alpha_{2}, \ldots, \alpha_{n}$ have already been defined, then we set

$$
\alpha_{n+1}(w)=\left.\left(\varphi-\sum_{i=1}^{n} \varphi_{\alpha_{i}}\right)\right|_{T_{n+1}}(w), \quad w \in T_{n+1} .
$$

Here $\varphi_{\alpha_{i}}$ are pseudocharacters introduced by equality (5) (note that $\varphi_{\alpha_{1}} \equiv 0$ ). Now we define the function $\alpha=\theta(\varphi)$ via its restriction to $T_{i}$ by setting $\left.\alpha\right|_{T_{i}}=\alpha_{i}$. Let us show that $\theta(\varphi)$ belongs to $L(T)$. Indeed, we have $\left.\alpha\right|_{T_{1}}=\alpha_{1} \equiv 0$. Furthermore, suppose that we have already established that the functions $\left.\alpha\right|_{T_{1}}, \ldots,\left.\alpha\right|_{T_{n}}$ are bounded. Let us prove that $\left.\alpha\right|_{T_{n+1}}$ is also bounded. Since the restrictions of the pseudocharacters $\varphi, \varphi_{\alpha_{2}}, \ldots, \varphi_{\alpha_{n}}$ to $T_{1}$ are zero functions it follows from Lemma 8 that all these pseudocharacters are bounded functions on $T_{n+1}$; therefore, formula (8) implies that the function $\left.\alpha\right|_{T_{n+1}}$ is bounded too.

Theorem 3. 1) The mapping $\theta$ is an isomorphism between the linear spaces $K$ and $L(T)$. The restriction of $\theta$ to $Z P X(\mathcal{F}, A)$ is an isomorphism between the linear spaces $Z P X(\mathcal{F}, A)$ and $L^{\prime}(T)$.

2) Each element $\varphi$ of the space $Z P X(\mathcal{F}, A)$ is uniquely representable in the form

$$
\varphi=\sum_{w \in T} \alpha(w) \bar{\delta}_{w}, \quad \alpha \in L^{\prime}(T) .
$$

Proof. Let us show that the mapping $\theta$ is linear. First we note that if $\tau_{1}, \tau_{2}, \tau$ are bounded functions on $T_{n+1}$ and $\lambda_{1}$ and $\lambda_{2}$ are reals such that $\tau=\lambda_{1} \tau_{1}+\lambda_{2} \tau_{2}$, then we have

$$
\varphi_{\tau}=\lambda_{1} \varphi_{\tau_{1}}+\lambda_{2} \varphi_{\tau_{2}},
$$

where $\varphi_{\tau}, \varphi_{\tau_{1}}, \varphi_{\tau_{2}}$ are the pseudocharacters defined by equality (5). Suppose that the function $\varphi \in K$ satisfies formula (8). Let $\lambda \in R$. Assume that we have already established that the restrictions of the functions $\theta(\lambda \varphi)$ to $T_{1}, \ldots, T_{n}$ are equal to $\lambda \alpha_{1}, \ldots, \lambda \alpha_{n}$, respectively. Then from formulas (8) and (9) we obtain $\left.\theta(\lambda \varphi)\right|_{T_{n+1}}=\left.\left(\lambda \varphi-\sum_{i=2}^{n} \varphi_{\lambda \alpha_{i}}\right)\right|_{T_{n+1}}=\left.\left(\lambda \varphi-\sum_{i=2}^{n} \lambda \varphi_{\alpha_{i}}\right)\right|_{T_{n+1}}=\lambda \alpha_{n+1}$, i.e., $\theta(\lambda \varphi)=\lambda \theta(\varphi)$. 
Let $\psi \in K$ and $\left.\theta(\psi)\right|_{T_{n}}=\beta_{n}, n \in N$. Then it is clear that $\left.(\varphi+\psi)\right|_{T_{1}} \equiv 0$ and $\left.(\varphi+\psi)\right|_{T_{2}}=\left.\varphi\right|_{T_{2}}+\left.\psi\right|_{T_{2}}=\alpha_{2}+\beta_{2}$. Suppose that we have already established that $\left.(\varphi+\psi)\right|_{T_{i}}=\left.\varphi\right|_{T_{i}}+\left.\psi\right|_{T_{i}}, i=1,2, \ldots, n$. Then for $n+1$ formulas (8) and (9) imply

$$
\begin{aligned}
\left.(\varphi+\psi)\right|_{T_{n+1}} & =\left.\left((\varphi+\psi)-\sum_{i=2}^{n} \varphi_{\left(\alpha_{i}+\beta_{i}\right)}\right)\right|_{T_{n+1}} \\
& =\left.\left((\varphi+\psi)-\sum_{i=2}^{n}\left(\varphi_{\alpha_{i}}+\varphi_{\beta_{i}}\right)\right)\right|_{T_{n+1}} \\
& =\left.\left(\varphi-\sum_{i=2}^{n} \varphi_{\alpha_{i}}\right)\right|_{T_{n+1}}+\left.\left(\psi-\sum_{i=2}^{n} \psi_{\beta_{i}}\right)\right|_{T_{n+1}} \\
& =\alpha_{n+1}+\beta_{n+1}=\left.\varphi\right|_{T_{n+1}}+\left.\psi\right|_{T_{n+1}} .
\end{aligned}
$$

Thus, the mapping $\theta$ is linear.

Let us show that $\theta$ maps $K$ onto $L(T)$. Indeed, let $\alpha \in L(T)$ and $\left.\alpha\right|_{T_{i}}=\alpha_{i}$. Since for each $t \in \mathcal{F}$ there is only a finite set of nonzero numbers of the form $\varphi_{\alpha_{n}}(t)$, $n \in \mathbb{N}$, the function $\varphi=\sum_{i=2}^{\infty} \varphi_{\alpha_{i}}$ on $\mathcal{F}$ is well defined and belongs to the space $K$. Let us show that $\theta(\varphi)=\alpha$. We set $\left.\theta(\varphi)\right|_{T_{i}}=\beta_{i}$; let us verify that $\beta_{i}=\alpha_{i}, \forall i \in N$. Since $\beta_{1}=\left.\varphi\right|_{T_{1}} \equiv 0$, we have $\beta_{1}=\alpha_{1}$. Suppose that we have already established the relations $\beta_{i}=\alpha_{i}$ for $i \leq n$; then for all $w \in T_{n+1}$ by formula (8) we have $\beta_{n+1}(w)=\left(\varphi-\sum_{i=2}^{n} \varphi_{\alpha_{i}}\right)(w)=\sum_{i=2}^{\infty} \varphi_{\alpha_{i}}(w)-\sum_{i=2}^{n} \varphi_{\alpha_{i}}(w)=\sum_{i=n+1}^{\infty} \varphi_{\alpha_{i}}(w)$. Furthermore, since $\left.\varphi_{\alpha_{i}}\right|_{T_{n+1}} \equiv 0, \forall i>n+1$, we obtain

$$
\sum_{i=n+1}^{\infty} \varphi_{\alpha_{i}}(w)=\varphi_{\alpha_{n+1}}(w)=\alpha_{n+1}(w)
$$

where the latter equality follows from Theorem 2 . Thus, $\beta_{n+1} \equiv \alpha_{n+1}$, and $\theta$ is an epimorphism.

Now let us verify that $\operatorname{ker} \theta=0$. Indeed, let $\varphi \in K$ and $\theta(\varphi) \equiv 0$. This means $\alpha_{i}=\left.\theta(\varphi)\right|_{T_{i}} \equiv 0, \forall i \in N$. Formula (8) implies $\left.\varphi\right|_{T_{1}} \equiv 0,\left.\varphi\right|_{T_{2}} \equiv 0$, and for each $w \in T_{n+1}$ we obtain $\varphi(w)=\sum_{i=2}^{n} \varphi_{\alpha_{i}}(w)+\alpha_{n+1}(w)=\sum_{i=2}^{n+1} \varphi_{\alpha_{i}}(w)$. Since $\alpha_{i} \equiv 0$, $\forall i \in \mathbb{N}$, we have $\varphi_{\alpha_{i}} \equiv 0$; therefore, the latter equality implies $\left.\varphi\right|_{T_{n+1}} \equiv 0$. Now from properties 1) and 2) of functions from the space $K$ it follows that $\varphi \equiv 0$ on $\mathcal{F}$. Therefore, $\operatorname{ker} \theta=0$ and $\theta$ is an isomorphism. Hence, as was shown above for a function $\alpha$ from $L(T)$ such that $\left.\alpha\right|_{T_{i}}=\alpha_{i}, \forall i \in N$, we have

$$
\theta^{-1}(\alpha)=\sum_{i=2}^{\infty} \varphi_{\alpha_{i}}
$$

Now let us show that under the isomorphism $\theta$ we assign to the elements of $Z P X(\mathcal{F}, A)$ functions $\alpha$ in $L(T)$ such that $\left|\int_{T} \alpha d \bar{\omega}_{x, y}^{A}\right| \leqslant \varepsilon$ for some $\varepsilon>0$ and each $x$ and $y$ in $\mathcal{F}$. Indeed, let $\varphi \in Z P X(\mathcal{F}), \alpha=\theta(\varphi)$, and $\alpha_{i}=\left.\alpha\right|_{T_{i}}, i \in N$; then formula (10) implies $\varphi=\sum_{i=2}^{\infty} \varphi_{\alpha_{i}}$. Now let us show that there exists an $\varepsilon$ such that $\left|\int_{T} \alpha d \bar{\omega}_{x, y}^{A}\right| \leqslant \varepsilon \quad$ for all $x$ and $y$ in $\mathcal{F}$. 
Let us choose $\varepsilon$ such that $|\varphi(x y)-\varphi(x)-\varphi(y)| \leqslant \varepsilon$ for each $x$ and $y$ in $\mathcal{F}$. Then we have

$$
\begin{aligned}
\left|\int_{T} \alpha d \bar{\omega}_{x, y}^{A}\right| & =\left|\sum_{i=2}^{\infty} \int_{T_{i}} \alpha d \bar{\omega}_{x, y}^{A}\right|=\left|\sum_{i=2}^{\infty} \int_{T_{i}} \alpha_{i} d \bar{\omega}_{x, y}^{A}\right| \\
& =\left|\sum_{i=2}^{\infty} \sum_{w \in T_{i}} \alpha_{i}(w) \bar{\omega}_{x, y}^{A}(w)\right| \\
& =\left|\sum_{i=2}^{\infty} \sum_{w \in T_{i}} \alpha_{i}(w)\left[\bar{\delta}_{w}(x y)-\bar{\delta}_{w}(x)-\bar{\delta}_{w}(y)\right]\right| \quad(\text { by Lemma } 7) \\
& =\left|\sum_{i=2}^{\infty}\left(\sum_{w \in T_{i}} \alpha_{i}(w) \bar{\delta}_{w}(x y)-\sum_{w \in T_{i}} \alpha_{i}(w) \bar{\delta}_{w}(x)-\sum_{w \in T_{i}} \alpha_{i}(w) \bar{\delta}_{w}(y)\right)\right| \\
& =\left|\sum_{i=2}^{\infty}\left(\varphi_{\alpha_{i}}(x y)-\varphi_{\alpha_{i}}(x)-\varphi_{\alpha_{i}}(y)\right)\right| \quad(\text { by Theorem 2) } \\
& =|\varphi(x y)-\varphi(x)-\varphi(y)| \leqslant \varepsilon .
\end{aligned}
$$

Now let $\alpha \in L(T)$ and $\left|\int_{T} \alpha d \bar{\omega}_{x, y}^{A}\right| \leqslant \varepsilon$ for some $\varepsilon>0$ and for each $x$ and $y$ from $\mathcal{F}$. If $\alpha_{i}=\left.\alpha\right|_{T_{i}}$ and $\varphi=\theta^{-1}(\alpha)$, then $\varphi=\sum_{i=2}^{\infty} \varphi_{\alpha_{i}}$. Therefore, we have

$$
\begin{aligned}
\mid \varphi(x y)- & \varphi(x)-\varphi(y)|=| \sum_{i=2}^{\infty}\left[\varphi_{\alpha_{i}}(x y)-\varphi_{\alpha_{i}}(x)-\varphi_{\alpha_{i}}(y)\right] \mid \\
= & \left|\sum_{i=2}^{\infty}\left[\sum_{w \in T_{i}} \alpha_{i}(w) \bar{\delta}_{w}(x y)-\sum_{w \in T_{i}} \alpha_{i}(w) \bar{\delta}_{w}(x)-\sum_{w \in T_{i}} \alpha_{i}(w) \bar{\delta}_{w}(y)\right]\right| \\
= & \left|\sum_{i=2}^{\infty} \sum_{w \in T_{i}} \alpha_{i}(w)\left[\delta_{w}(x y)-\bar{\delta}_{w}(x)-\bar{\delta}_{w}(y)\right]\right| \\
& =\left|\sum_{i=2}^{\infty} \sum_{w \in T_{i}} \alpha_{i}(w) \bar{\omega}_{x, y}^{A}(w)\right|=\left|\int_{T} \alpha(w) d \bar{\omega}_{x, y}^{A}(w)\right| \leqslant \varepsilon .
\end{aligned}
$$

The theorem is proved.

Denote by $R^{T_{1}}$ the space of real-valued functions on the set $T_{1}$.

Lemma 9. The expression $\sum_{t \in T_{1}} \gamma(t) \bar{\delta}_{t}$ is the general form of an element from the space $X(\mathcal{F}, A)$.

Proof. Follows from Lemmas 5 and 6.

It is evident that the mapping $\gamma \rightarrow \sum_{t \in T_{1}} \gamma(t) \bar{\delta}_{t}$ is an isomorphism between the linear spaces $R^{T_{1}}$ and $X(\mathcal{F}, A)$.

Corollary 1. Each element $f$ of the space $\operatorname{PX}(\mathcal{F}, A)$ is uniquely representable in the form

$$
f=\chi+\sum_{w \in T} \alpha(w) \bar{\delta}_{w}, \quad \chi \in X(\mathcal{F}, A), \quad \alpha \in L^{\prime}(T) .
$$

Proof. Let $\chi$ be an element of $X(\mathcal{F})$ such that $\chi(x)=f(x) \quad \forall x \in X$. It is easy to see that $\chi \in X(\mathcal{F}, A)$. Then we have $f-\chi \in Z P X(\mathcal{F}, A)$. Now from Theorem 3 we obtain the required representation. 
Corollary 2. Let $\mathcal{F}$ be a nonabelian free semigroup and let $S=B \cdot \mathcal{F}$ be the semidirect product of the semigroups $B$ and $\mathcal{F}$, where $B$ acts on $\mathcal{F}$ by automorphisms. Then there is a nontrivial pseudocharacter on the semigroup $S$. (We say that a pseudocharacter $\varphi$ is nontrivial if it is not an additive real character.)

Proof. Let $w=x_{1} x_{2}$, where $x_{1}, x_{2} \in X$. It is easy to see that $\delta_{w}$ is not a character and belongs to the space $P X(\mathcal{F}, A u t \mathcal{F})$. Taking into account Theorem 1 and the relation $P X(\mathcal{F}, A u t \mathcal{F}) \subseteq P X(\mathcal{F}, B)$, we obtain $\delta_{w} \in P X(S)$.

\section{ACKNOWLEDGEMENT}

The author is indebted to the referee for emphasizing the connection of the results of the paper with the theory of Banach algebra cohomology.

\section{REFERENCES}

1. Ulam, S. M., A Collection of Mathematical Problems, Wiley \& Sons, Interscience Publ., New York-London, 1960. MR 22:10884

2. Hyers, D. H., On the stability of the linear functional equation, Proc. Nat. Acad. Sci. USA 27 (2) (1941), 222-224. MR 2:315a

3. Hyers, D. H., Ulam, S. M., On approximate isometry, Bull. Amer. Math. Soc. 51 (1945), 288-292. MR 7:123f

4. Hyers, D. H., Ulam, S. M., Approximate isometries of the space of continuous functions, Ann. of Math. 48 (2) (1947), 285-289. MR 8:588b

5. Baker, J., Lawrence, J., Zorzitto, F., The stability of the equation $f(x+y)=f(x)+f(y)$, Proc. Amer. Math. Soc. 74 (2) (1979), 242-246. MR 80d:39009

6. Shtern, A.I., Stability of homomorphisms into the group $R^{*}$, Vestnik MGU, Ser. matem. mekh. 37 (3) (1982), 33-36. MR 83m:39008

7. Grove, K., Karcher, H., Ruh, E. A., Jacobi fields and Finsler metrics on compact Lie groups with an application to differential pinching problems, Math. Ann. 211 (1) (1974), 7-21. MR 50:8391

8. de la Harpe, P., Karoubi, M., Represéntations approchées d'un groupe dans une algèbre de Banach, Manuscripta math. 22 (3) (1977), 297-310. MR 58:16948

9. Kazhdan, D., On ع-representations, Israel J. Math. 43 (4) (1982), 315-323. MR 84h:22011

10. Shtern, A. I., A pseudocharacter that is determined by the Rademacher symbol, Uspekhi mat. nauk 45 (3) (1990), 224-226. MR 91j:11027

11. Shtern, A. I., Quasirepresentations and pseudorepresentations, Funkts. analiz i eqo prilozhen. 25 (2) (1991), 70-73. MR 93b:22007

12. Faïiev, V.A., Pseudocharacters of free products of semigroups, Funkts.analiz. i ego priloz. 21 (1) (1987), 86-87. MR 88e:20054

13. Faïziev, V.A., Pseudocharacters on free groups and on some group constructions, Uspehi mat. nauk. 43 (5) (1988), 225-226. MR 90a:20047

14. Fä̈iev, V.A., Spaces of pseudocharacters of the free product of semigroups, Mathem.Zametki 52 (6) (1992), 119-130. MR 93m:20087

15. Faiziev, V.A., Pseudocharacters on semidirect product of semigroups, Mathem.Zametki 53 (2) (1993), 132-139. MR 94h:20070

16. Faiziev, V.A., Pseudocharacters on free group, Izvestya Russ. Akad. Nauk ser. mathem. 58 (1) (1994), 121-143. MR 95b:20041

17. Fă̈ziev, V.A., Pseudocharacters on free semigroups, Russian Journal of Math. phisics 3 (2) (1995), 191-206. MR 96h:20108

18. Johnson B.E., Cohomology in Banach algebras, Mem. Amer. Math. Soc. 127 (1972). MR 51:11130

Institute for Mathematics with Computational Center, Tadjikistan Academy of Sciences, Dushanbe, Tadjikistan

Current address: Shirokaia St. 7-3-137, 129282 Moscow, Russia 